\title{
A COMPARATIVE STUDY OF SUB-10NM SI, GE AND GAAS N-CHANNEL FINFET
}

\section{SHAFIQUL ISLAM ${ }^{1}$, SHARIF UDDIN ${ }^{2}$, HASAN ALI ${ }^{3}$, JAVED HOSSAIN $^{4} \&$ ZAHID HASAN MAHMUD ${ }^{5}$}

1, 2, 3 Department of Electronics and Telecommunication Engineering, Begum Rokeya University, Rangpur, Bangladesh

${ }^{4}$ Department of Computer Science and Telecommunication Engineering, Noakhali Science and

Technology University, Noakhali, Bangladesh

${ }^{5}$ Department of Electrical and Electronic Engineering, University of Dhaka, Dhaka, Bangladesh

ABSTRACT
In this paper, we explored the performance variability of FinFET for doped/ undoped channels, with different
gate materials. Multi-gate FETs such as FinFETs and double gate FETs enable the CMOS technology, to scale beyond
sub-22nm node. Following the current trends, we present a comparative study of double gate n-FinFET structures
considering different parameters for Si, Ge and GaAs channel parameters. The self-consistent solutions of Poisson and
validate the superiority of GaAs, compared to other considered materials.
KEYWORDS: DIBL, Transconductance, Subthreshold Swing(SS) \& Finfet

Received: Sep 30 2017; Accepted: Oct 20, 2017; Published: Nov 11, 2017; Paper Id.: IJSSTDEC20171

\section{INTRODUCTION}

The planar bulk Si metal-metal oxide semiconductor field effect transistor (MOSFET) has been reached its scaling limit. To extend its limit as well as its performance, different new materials and different device architectures have been introduced in the complementary MOS (CMOS) technologies. In this connection, polycrystalline $\mathrm{Si}$ electrode $/ \mathrm{SiO}_{2}$ has been replaced by high-k dielectric with metallic electrode. [I] Moreover as a part of the development of CMOS technology, for reducing power consumption without loss of speed, we are going to replace the core material of MOSFET, the Si channel. And we replace this by high mobility semiconductor such as germanium or III-V compound semiconductor.[II]

Recently we found a remarkable improvement of understanding of high-k III-V interfaces. III-V FinFETs as well as multi -gate quantum well FET have been shown to improve greatly because this device shows reduce short channel effect(SCE) higher current drivability, nearly ideal sub threshold swing and mobility enhancement. The most important development is cost-effective fabrication method to integrate highly crystalline non-Si channel material on a Si wafer.[II]

Again $\mathrm{Ge}$ has the highest hole mobility among $\mathrm{Si}$, Ge and $\mathrm{GaAs}$ and greater electron mobility than $\mathrm{Si}, \mathrm{Ge}$ is the most promising material to replace $\mathrm{Si}$ as the channel material for future technology [III]. On the other hand $\mathrm{Ge}$ have $4.2 \%$ lattice mismatch than $\mathrm{Si}$, resulting Threading Dislocation Density (TDD) values typically in order of $108-109 \mathrm{~cm}^{3}$. This is not acceptable for most application.[I]

Within this context, we consider $\mathrm{Si}, \mathrm{Ge}$ and GaAs respectively as the channel material of $\mathrm{n}$-channel 
FinFET for performance evaluation. First, we study the different characteristic like Tran conductance, sub-Threshold swing, DIBL and many more. Then, we present a comparative analysis among them and finally draw a comprehensive conclusion based on obtained results.

\section{DEVICE STRUCTURE AND DIMENSIONS}

The device structure of double gate n-channel FinFET structure has been illustrated in Fig 1. Figure 1 consist of gate length also called channel length is indicated by $L_{g}$ and channel width $W_{c h}$. A oxide layer is placed on either side of the channel and also, at the top surface of the channel. The thickness of the side wall oxide is specified by $\mathrm{T}_{\mathrm{ox} 1}$ and $\mathrm{T}_{\mathrm{ox} 2}$. For the present simulation, we use $2 \mathrm{~nm}$ thickness for oxide layer. Here, thickness of Channel width is $10 \mathrm{~nm}$. Gate length is $45 \mathrm{~nm}$ and for both source extension and drain extension length is 50nm length. In both case, gate overlap to source and drain of $2 \mathrm{~nm}$ in length. The drain/source doping has been kept fixed at $1 \times 10^{20} \mathrm{~cm}^{3}$ and channel $1 \times 10^{16} \mathrm{~cm}^{3}$. The Drain bias has been taken $0.05 \mathrm{~V}$ and $1 \mathrm{~V}$, gate bias varied from $0 \mathrm{~V}$ to $1 \mathrm{~V}$ and we have chosen different physical constant for different materials [IV]

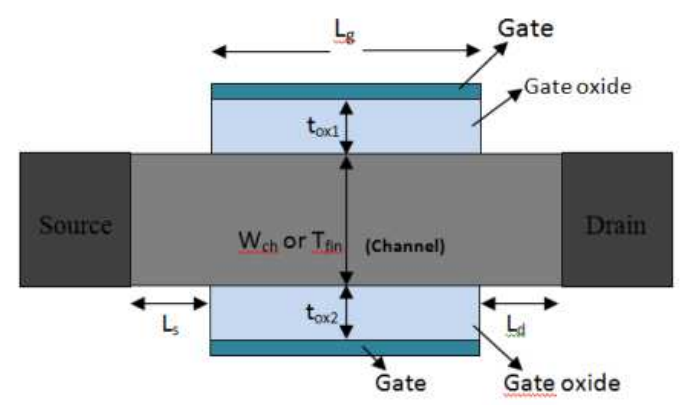

Figure1: Two dimensional Double-Gate Finfet[IX]

The presented results are based on drift-diffusion model [V]. The model has been used in the present calculations because of the fact that sub-threshold characteristics of these devices are diffusion dominated and reflects device characteristics in the sub-threshold region well in consistence with the experimentally observed results [V] [VI][VII].

\section{SIMULATION AND RESULTS}

To study the characteristics variation of Multigate FinFET, we use MuGFET tool, which uses drift-diffusion type simulator PROPHET and PADRE. This simulator is provided by NanoHub. Org, developed at BELL LAB. It cannot choose Quantum transport simulation, which is very expensive and difficult to include all the scattering processes. The drift diffusion type simulator works well enough, to demonstrate characteristics of relatively long and large devices. PROPHET and PADRE are self-consistent simulator. PROPHET can solve Poisson equation, drift diffusion equation and PADRE energy balance equation [VIII].

\section{Gate Voltage Vs Drain Current}

In order to study the drain current with respect to the gate voltage, we found different data(shown in table 1) using PADRE simulator. Here we have taken different values of drain current for two different drain bias point valued $0.05 \mathrm{~V}$ and $1.00 \mathrm{~V}$. 
Table 1: Variation of Drain Current With Respect to Gate Voltage For Si, Ge and GaAs

\begin{tabular}{|c|c|c|c|c|c|c|}
\hline & \multicolumn{2}{|c|}{ Si } & \multicolumn{2}{c|}{ Ge } & \multicolumn{2}{c|}{ Gaas } \\
\hline $\begin{array}{c}\text { Gate } \\
\text { Voltage } \\
\text { (V) }\end{array}$ & $\begin{array}{c}\text { Drain } \\
\text { Current } \\
\text { for } \\
\text { Vd=.05v }\end{array}$ & $\begin{array}{c}\text { Drain } \\
\text { Current } \\
\text { for } \\
\text { Vd=1 }\end{array}$ & $\begin{array}{c}\text { Drain } \\
\text { Current } \\
\text { for } \\
\text { Vd=.05v }\end{array}$ & $\begin{array}{c}\text { Drain } \\
\text { Current } \\
\text { for } \\
\text { Vd=1v }\end{array}$ & $\begin{array}{c}\text { Drain } \\
\text { Current } \\
\text { for } \\
\text { Vd=.05v }\end{array}$ & $\begin{array}{c}\text { Drain } \\
\text { Current } \\
\text { for } \\
\text { Vd=1v }\end{array}$ \\
\hline 0 & $5.43 \mathrm{E}-12$ & $8.25 \mathrm{E}-12$ & $2.55 \mathrm{E}-12$ & $3.86 \mathrm{E}-12$ & $1.20 \mathrm{E}-11$ & $1.86 \mathrm{E}-11$ \\
\hline 0.1 & $2.19 \mathrm{E}-10$ & $3.38 \mathrm{E}-10$ & $6.54 \mathrm{E}-11$ & $1.16 \mathrm{E}-10$ & $4.84 \mathrm{E}-10$ & $7.54 \mathrm{E}-10$ \\
\hline 0.3 & $3.19 \mathrm{E}-07$ & $4.99 \mathrm{E}-07$ & $8.74 \mathrm{E}-08$ & $1.58 \mathrm{E}-07$ & $7.06 \mathrm{E}-07$ & $1.07 \mathrm{E}-06$ \\
\hline 0.5 & $5.06 \mathrm{E}-05$ & $7.69 \mathrm{E}-05$ & $4.17 \mathrm{E}-05$ & $5.52 \mathrm{E}-05$ & $9.98 \mathrm{E}-05$ & 0.00011 \\
\hline 0.7 & $7.38 \mathrm{E}-05$ & 0.00031 & 0.00013 & 0.00034 & 0.00021 & 0.00035 \\
\hline 0.9 & $7.60 \mathrm{E}-05$ & 0.00055 & 0.00014 & 0.00064 & 0.00021 & 0.00056 \\
\hline 1 & $7.64 \mathrm{E}-05$ & 0.00066 & 0.00014 & 0.00078 & 0.00021 & 0.00065 \\
\hline
\end{tabular}

The variation of Drain Current with gate voltage for two bias point has been plotted in Figure-2

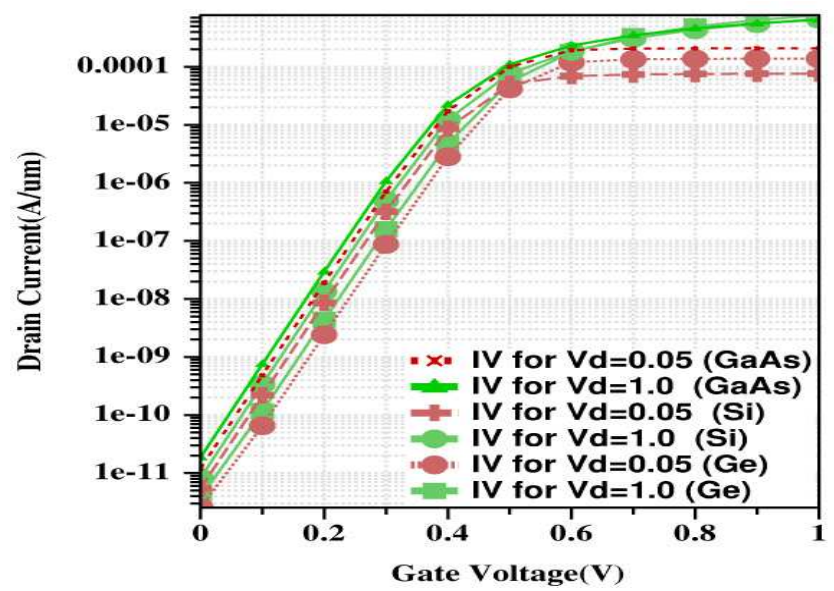

Figure 2: Gate Voltage V/S Drain Current

\section{Drain Voltage Vs Transconductance}

Trans conductance is the electrical characteristic relating the current through the output of a device to the voltage across the input of a device. Transconductance is the reciprocal of resistance. For an FET transconductance is the ratio of the change in drain current to the change in gate voltage.

Here we used PADRE simulator for two bias point $0.05 \mathrm{~V}$ and $1.0 \mathrm{~V}$. We have found different values of transconductance for $\mathrm{Si}, \mathrm{Ge}$ and $\mathrm{GaAs}$ which are plotted and found the following curve.

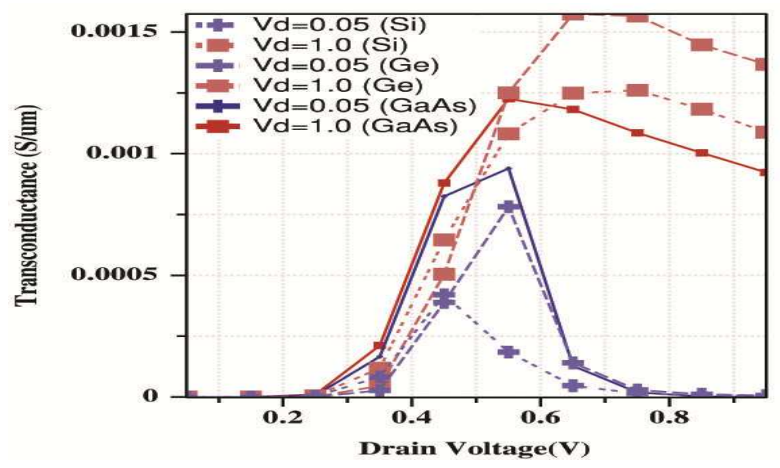

Figure 3: Drain Voltage V/S Transconductance 


\section{Subthreshold Swing Vs Drain Voltage}

In case of studying the SS characteristics with respect to Drain voltage. We have considered different types of drain biasing which are shown in table 2 .

Table 2: Variation of SS With Respect to Different Bising Point of Drain Voltage for $\mathrm{Si}$, Ge, and GaAs

\begin{tabular}{|c|c|c|c|}
\hline \multirow{2}{*}{ Drain Voltage(V) } & \multicolumn{3}{|c|}{ Ss(Mv/Dec) } \\
\cline { 2 - 4 } & $\mathbf{S i}$ & $\mathbf{G e}$ & Gaas \\
\hline 0.0500 & 64.6092 & 66.2821 & 65.1752 \\
\hline 0.1857 & 80.0006 & 83.4317 & 80.8254 \\
\hline 0.3214 & 80.0146 & 83.1057 & 80.9898 \\
\hline 0.4571 & 80.0861 & 82.9076 & 83.5350 \\
\hline 0.5929 & 80.1797 & 82.7811 & 83.5851 \\
\hline 0.7286 & 80.2806 & 82.6983 & 83.6476 \\
\hline 0.8643 & 80.3828 & 82.6446 & 83.7159 \\
\hline
\end{tabular}

Using these values we have plotted Subthreshold swing, with respect to drain voltage for Si, Ge and GaAs, which are shown in figure-4.

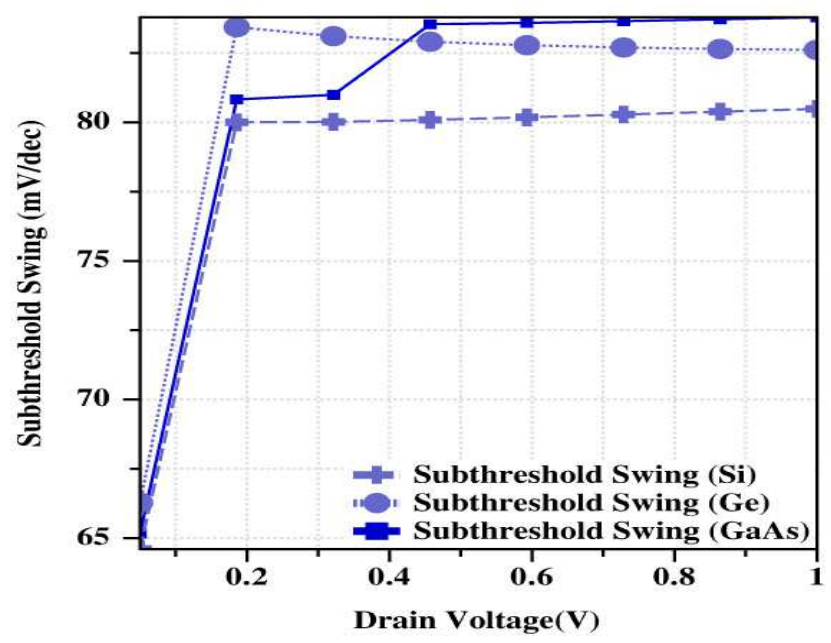

Figure.4: SS V/S Drain Voltage

\section{DIBL Vs Drain Voltage}

In case of studying the DIBL characteristics with respect to Drain voltage. we have consider different types of drain biasing point which are shown in Table 3) Drain induced Barrier Lowering value with respect to drain voltage for Si, Ge and GaAs.

Table 3: Variation of DIBL with Respect to Drain Voltage for Si, Ge and GaAs

\begin{tabular}{|c|c|c|c|}
\hline \multirow{2}{*}{ Drain Voltage(V) } & \multicolumn{3}{|c|}{ Dibl(Mv/V) } \\
\cline { 2 - 4 } & $\mathbf{S i}$ & Ge & Gaas \\
\hline 0.186 & --- & 164.106 & 17.997 \\
\hline 0.321 & 33.226 & 23.157 & 8.736 \\
\hline 0.457 & 18.982 & 15.561 & 5.926 \\
\hline 0.593 & 13.017 & 11.854 & 4.558 \\
\hline 0.729 & 10.011 & 9.760 & 3.769 \\
\hline 0.864 & 8.218 & 8.410 & 3.262 \\
\hline 1.000 & 7.035 & 7.445 & 2.895 \\
\hline
\end{tabular}


After Plotting these values we found the following curve. Which is shown in figure 5.

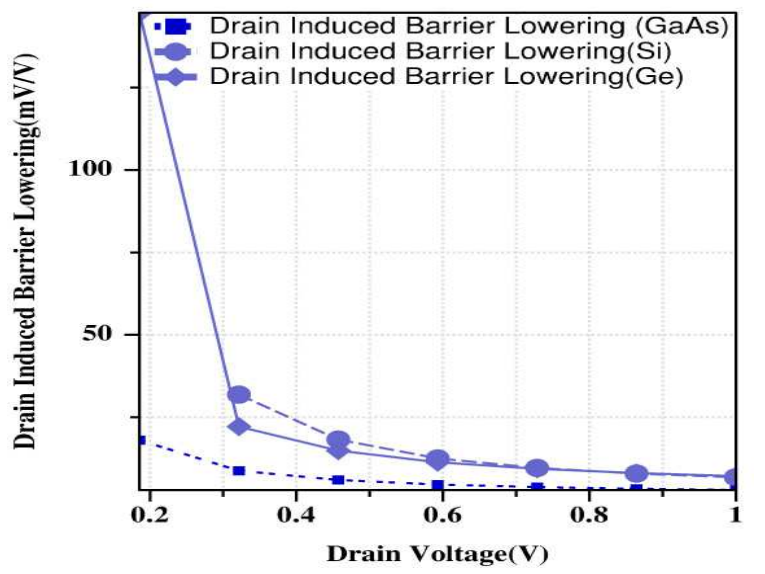

Figure 5: DIBL V/S Drain Voltage for Si, Ge and GaAs

\section{CONCLUSIONS}

In this paper I-V, DIBL, SS and transconductance characteristics has been carried out for a FinFET structure, utilizing different channel material at every time. The DIBL variation, with respect to drain voltage of GaAs show better performance, than other two silicon and germanium channel materials FinFET. The dominance performance of GaAs continues for subthreshold swing, with respect to drain voltage. Analysis of all the I-V characteristics, again shows the leading performance compared to others. Due to the above mentioned advantages, the use of GaAs as channel material could show more resistance, against short channel effect, compared to others. Analysis of transconductance, with respect to drain voltage shows a peak in the transconductance turn, on bias between $\mathrm{Vd}=0.6 \mathrm{~V}$ to $\mathrm{Vd}=0.8 \mathrm{~V}$. The flat transconductance is largely invariant to most process variations, but spacing between the wrapped gate must be chosen, so that the transconductance remain flat and at a high magnitude.

\section{REFERENCES}

1. M. J. H. Van Dal et al., “Germanium p-Channel FinFET Fabricated by aspect ratio trapping," IEEE Trans. Electron Devices, vol. 61, no. 2, pp. 430-436, 2014

2. S. L. Tripathi, RamanujMishra and R. A. Mishra "Characteristic comparison of connected DG FINFET, TG FINFET and Independent Gate FINFET on $32 \mathrm{~nm}$ technology" 2012, 2nd International Conference on Power, Control and mbedded Systems.

3. B. Duriez, G. Vellianitis, M. J. H. van Dal, G. Doornbos, R. Oxland, K. K. Bhuwalka, M. Holland, Y. S. Chang, C. H. Hsieh, K. M. Yin, Y. C. See, M. Passlack, C. H. Diaz "Scaled p-channel Ge FinFET with optimized gate stack and record performance integrated on $300 \mathrm{~mm}$ Si wafers.

4. https://nanohub. org.

5. T. A. Bhat, M. Mustafa, and M. R. Beigh, “Vol. 7 No 3, 03010(5pp) (2015) Ж,” vol. 7, no. 3, pp. 1-5, 2015.

6. Tai-su Park, Euijoon Yoon, Jong-Ho Lee, Physica E 19, 6 (2003).

7. www. ioffe. ru

8. N. State, “First-Time User Guide,” pp. 1-33, 2008. 
9. H. Shang et al., "Investigation of FinFET Devices for 32nm Technologies and Beyond,” 2006 Symp. VLSI Technol. 2006. Dig. Tech. Pap., no. 914, pp. 2005-2006, 2006.

10. J. J. Gu, Y. Q. Liu, Y. Q. Wu, R. Colby, R. G. Gordon, and P. D. Ye, "First experimental demonstration of gate-all-around III\&\#x2013;V MOSFETS by top-down approach,” 2011 Int. Electron Devices Meet., no. 100, p. 33.2.1-33.2.4, 2011.

11. Tawseef A. Bhat, M. Mustafa, M. R. Beigh, "Study of Short Channel Effects in n-FinFET tructure for Si, GaAs, GaSb and GaN Channel Materials” JOURNAL OF NANO AND ELECTRONIC PHYSICS Vol. 7 No 3, 03010(5pp) (2015) 